\title{
2D Compass Codes
}

\author{
Muyuan Li, ${ }^{1}$ Daniel Miller, ${ }^{2}$ Michael Newman, ${ }^{3, *}$ Yukai $\mathrm{Wu},{ }^{4}$ and Kenneth R. Brown ${ }^{3, \dagger}$ \\ ${ }^{1}$ School of Computational Science and Engineering, Georgia Institute of Technology, \\ Atlanta, Georgia 30332, USA \\ ${ }^{2}$ Institut für Theoretische Physik III, Heinrich-Heine-Universität Düsseldorf, \\ D-40225 Düsseldorf, Germany \\ ${ }^{3}$ Departments of Electrical and Computer Engineering, Chemistry, and Physics, Duke University, \\ Durham, North Carolina 27708, USA \\ ${ }^{4}$ Department of Physics, University of Michigan, Ann Arbor, Michigan 48109, USA
}

(Received 26 September 2018; revised manuscript received 5 March 2019; published 29 May 2019; corrected 17 March 2020)

\begin{abstract}
The compass model on a square lattice provides a natural template for building subsystem stabilizer codes. The surface code and the Bacon-Shor code represent two extremes of possible codes depending on how many gauge qubits are fixed. We explore threshold behavior in this broad class of local codes by trading locality for asymmetry and gauge degrees of freedom for stabilizer syndrome information. We analyze these codes with asymmetric and spatially inhomogeneous Pauli noise in the code capacity and phenomenological models. In these idealized settings, we observe considerably higher thresholds against asymmetric noise. At the circuit level, these codes inherit the bare-ancilla fault tolerance of the Bacon-Shor code.
\end{abstract}

DOI: 10.1103/PhysRevX.9.021041

Subject Areas: Quantum Information

\section{INTRODUCTION}

At the heart of scalable quantum computing is fault tolerance [1-3]. To optimize the fault tolerance of a system, one should adapt the information extracted from the system to the particular noise it suffers from. Typical models of fault tolerance assume isotropic error that is homogeneous in space. This has resulted in the broad study of codes that are invariant over space with unbiased noise. We study a family of codes derived from the compass model of spin interactions that can be tailored to specific noise models and maintain simple fault-tolerance procedures. We find that this tailoring can greatly improve the fault tolerance of a system in idealized settings, reinforcing the perspective that noise asymmetry is a resource that should be taken advantage of. Combined with other methods that benefit from asymmetric noise [4,5], our results point towards methods for deriving other families of subspace codes from subsystem codes.

Our focus is on codes built from local interactions. For physical systems that prefer such interactions, topological codes have emerged as leading candidates for fault-tolerant

\footnotetext{
*michael.newman@duke.edu

kenneth.r.brown@duke.edu
}

Published by the American Physical Society under the terms of the Creative Commons Attribution 4.0 International license. Further distribution of this work must maintain attribution to the author(s) and the published article's title, journal citation, and DOI. quantum computation [6-13]. Among these, the surface code is a particularly enticing choice, offering depolarization accuracy thresholds in excess of $15 \%$, assuming efficient, noiseless, error correction with a planar architecture [14].

Another code family that has generated significant interest is the subsystem Bacon-Shor code family [15]. These codes have many desirable properties: Their gauge group is 2-local, measurements can be performed with bare ancilla with virtually no loss in performance $[16,17]$, and they support fault-tolerance schemes that avoid costly magic-state distillation [18]. Unfortunately, while BaconShor codes offer some of the highest concatenated thresholds [16], they fail to have any threshold when grown as a local family on a lattice without concatenation [19].

In the present article, we investigate codes derived from the quantum compass model on a square lattice [20]. This model provides a natural framework for constructing subsystem and subspace stabilizer codes. These codes can be viewed as different gauge fixes of the Bacon-Shor code and so include the (rotated) surface codes, as well as codes with certain topological defects, as members $[8,9]$. While we focus on a subfamily of (generalized) surface codes [21] with desirable fault-tolerance properties, the design space for these codes is much larger. Two advantages of this family are its malleability, making it suitable for correcting asymmetric noise, and fault-tolerant bare-ancilla syndrome extraction inherited from measuring along the gauges of the template Bacon-Shor code.

Tailoring codes and decoders to specific noise models can often yield fruitful improvements in threshold scaling. 
For biased noise models, one can choose fault-tolerance schemes and gates that take advantage of asymmetric error rates [22-24]. Indeed, simply choosing the right decoder can yield tremendous gains in the effective threshold [5,25-28]. One can even customize codes directly to device-level noise [29] or biased error rates [4,30]. Such asymmetric noise models are motivated experimentally by the observation that dephasing noise dominates certain quantum computing architectures [31]. By modifying the stabilizers and boundaries of a planar code directly, one can also obtain denser packings of logical qubits [21] and optimized performance with respect to erasures [32].

We similarly modify the geometry of planar codes using the convenient language of compass codes, adapting the density of the syndrome information to better correct biased and spatially dependent Pauli noise. To quantify the value of this adapted syndrome data, we consider maximumlikelihood [6,33], minimum-weight perfect matching [6,14] and union-find [34] decoders that treat $X$ - and $Z$-type errors independently. We choose different decoders depending on the context, but we generally observe similar performance across all three. In particular, we expect that tuning correlated decoders to account for these different noise models will boost code performance even further [5].

The idea is simple: One should tessellate a lattice according to the relative likelihood of errors in that part of the lattice. Although these codes remain local, there is a trade-off between the locality of their stabilizers and their robustness against asymmetric noise, similar to Ref. [24]. We analyze these codes numerically in the code capacity and phenomenological noise models, and observe considerably higher thresholds against asymmetric noise in these idealized settings. We leave a discussion of the challenges posed by circuit-level noise to the conclusion.

The paper is structured as follows. In Sec. II, we introduce 2D compass codes and the noise models we consider. In Sec. III, we determine the threshold behavior in two randomized families of codes interpolating between Bacon-Shor codes, surface codes, and Shor's code. In Sec. IV, we quantify the threshold of 2D compass codes tailored for different asymmetric noise models. In Sec. V, we demonstrate fault tolerance for the compass-code family using only bare-ancilla syndrome extraction. We conclude with some discussion in Sec. VI.

\section{BACKGROUND}

We begin by defining a subspace stabilizer code, which is an encoding of logical information into subspace $C$ of an ambient Hilbert space $\mathcal{H}$,

$$
\mathcal{H}=C \oplus C^{\perp},
$$

where $C$ can be defined as the +1 -eigenspace of $n-k$ commuting Pauli operators generating the stabilizer group. In this case, we say that the code has parameters $n$, the $\log$ dimension of $\mathcal{H}$, and $k$, the $\log$ dimension of $C$. Informally, this represents an encoding of $k$ qubits into $n$ qubits.

A subsystem stabilizer code is a generalization of a subspace stabilizer code in which we allow some number $g<k$ of the logical degrees of freedom (d.o.f.) to vary. In this case, we further decompose our space as

$$
\mathcal{H}=(\mathcal{L} \otimes G) \oplus C^{\perp},
$$

where $g$ is the $\log$ dimension of $G$ and $k-g$ is the $\log$ dimension of $\mathcal{L}$. We call the group generated by all logical Pauli operators acting trivially on $\mathcal{L}$ the gauge group of the code, which has the stabilizer group of the underlying subspace code as its center. This broad class encompasses the majority of popular code families, where subspace codes are the special case of $g=0$.

\section{A. 2D compass codes}

The quantum compass model on a square lattice is generally defined by the Hamiltonian $[35,36]$

$$
H=\sum_{i \neq L-1} \sum_{j} J_{X} X_{i, j} X_{i+1, j}+\sum_{i} \sum_{j \neq L-1} J_{Z} Z_{i, j} Z_{i, j+1} .
$$

Here, $(i, j)$ indexes a qubit according to its displacement from the top-left corner of the lattice. Closely connected with this model are Bacon-Shor codes, which are stabilizer subsystem codes with gauge operators realized by the twobody interaction terms of the compass model [15]. This family is a standard example of codes requiring only local measurements for error correction, but which are not topological, with stabilizers that extend the length of the lattice.

The gauge group of a Bacon-Shor code is generated by $\mathcal{G}=\left\langle X_{i, j} X_{i+1, j}, Z_{i, j} Z_{i, j+1}\right\rangle$, with stabilizer group $\mathcal{S}=$ $\left\langle\prod_{j} X_{i, j} X_{i+1, j}, \prod_{i} Z_{i, j} Z_{i, j+1}\right\rangle$. When defined on an $L \times L$ lattice, these $2 L$-body stabilizer generators leave us with $(L-1)^{2}$ gauge d.o.f. to format as we please.

Our tool for constructing compass codes will be the method of gauge fixing, by which we can insert gauge transformations into the stabilizer group [7,37]. Operationally, up to phase, this method corresponds to inserting a gauge operator $g$ into $\mathcal{S}$ and then removing the set of all gauge operators $h$ that anticommute with $g$ from $\mathcal{G}$. Note that as Bacon-Shor codes are CSS codes [38,39], if we perform fixes of either $X$ or $Z$ type, we preserve the CSS structure.

We focus on a subclass of surface codes that are easy to specify via a coloring of the lattice (see Fig. 1). In that graphical language, red plaquettes correspond to "cuts" in the vertical $Z$-type stabilizers. By this, we mean that the $Z$-type stabilizer is divided into two independent $Z$ stabilizers by the red plaquette, extending the stabilizer group by one generator while expending 1 gauge d.o.f. In particular, the original Bacon-Shor code stabilizers remain in the stabilizer group of any compass code. 


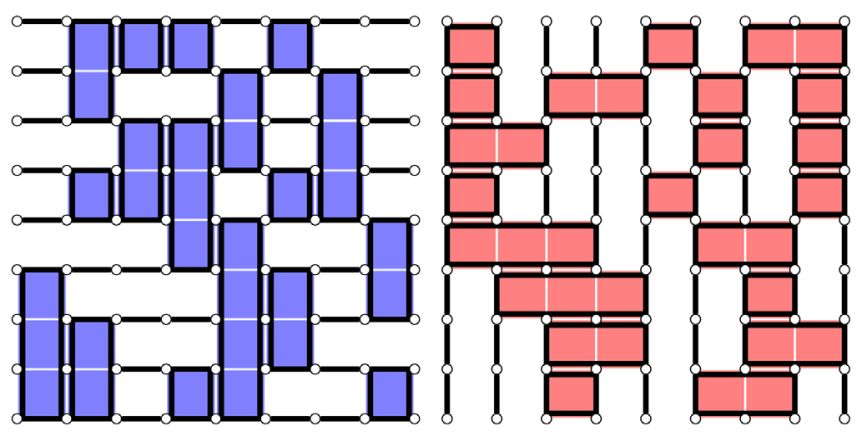

FIG. 1. An example of a compass code on a $9 \times 9$ lattice with qubits placed on vertices. Red and blue plaquettes represent cuts in the $Z$-type and $X$-type stabilizers, respectively. Equivalently, $Z$-type stabilizers are formed by blue plaquettes on the left, and $X$-type stabilizers are formed by red plaquettes on the right. Bare horizontal lines on the left represent horizontal $Z Z$ stabilizers or "0"-area blue plaquettes; the analogous vertical lines on the right form $X X$ stabilizers. As there are no blank plaquettes in the combined picture, all of the gauge d.o.f. are fixed.

We index plaquettes according to the index of their topleft qubit; then, for a red plaquette in the $(i, j)$ th cell of the lattice, we fix the gauge operator $\prod_{k=0}^{i} Z_{k, j} Z_{k, j+1}$, whereas for a blue plaquette, we fix $\prod_{k=0}^{j} X_{i, k} X_{i+1, k}$. Bacon-Shor codes correspond to an empty coloring, whereas the standard surface code corresponds to a red and blue checkerboard. Note that a plaquette can be colored either red or blue, but not both, as the resulting stabilizers would not commute.

\section{B. Noise models}

In order to carry out numerical simulations, we restrict ourselves to asymmetric Pauli noise. We consider the $\eta$-biased depolarizing channel with error rate $p$, defined as

$$
\mathcal{E}(\rho)=(1-p) \rho+p_{X} X \rho X+p_{Y} Y \rho Y+p_{Z} Z \rho Z,
$$

where $p=p_{X}+p_{Y}+p_{Z}$ and $\eta=p_{Z} /\left(p_{X}+p_{Y}\right)$. We make the simplifying assumption that $p_{X}=p_{Y}$, matching the definition in Ref. [5]. The notion of a physical error rate $p$ is then well defined, as the fidelity of such a channel to the identity is independent of $\eta$.

We consider symmetric but biased noise, in which each qubit experiences the same error channel, as well as spatially inhomogeneous noise models, in which the error channel may depend on the qubit's position in the lattice. In the latter case, we define the error rate and bias of the channel on the lattice as a whole as the average fidelity and bias over each qubit in the lattice. Note that we must be careful in comparing such models. For example, concentrating noise on a small subset of qubits might always produce perfectly correctable errors, whereas distributing that noise symmetrically will not.
Finally, depending on the context, we consider either the code capacity setting, in which syndrome measurements are assumed to be perfect, or the phenomenological setting, in which the syndrome measurements can be faulty.

\section{Decoders}

Inherent to any discussion on thresholds is a choice of decoder. We focus on three decoders: independent maximum-likelihood, minimum-weight perfect matching, and union-find decoding. We choose among these according to our computational needs.

Each of these decoders corrects $X$ - and Z-type errors independently. Thus, any gains in threshold scaling are a product of the tailored syndrome information alone; it is these gains we aim to quantify. For example, we expect that using a correlated decoder with $X$ - and $Y$-type stabilizers would augment the threshold further [5].

\section{Decoder graph}

For independent correction of $X$ - and $Z$-type errors on a CSS code, the relevant decoding information is captured in the decoder (hyper)graph. The decoder graph for phase errors is constructed by associating a vertex to each $X$-type stabilizer and a (hyper)edge to each qubit, where the edges connect all stabilizers incident to that qubit. The decoder graph for bit-flip errors is defined analogously. Note that for the subspace codes we consider, the decoder graph corresponds to a cellulation which realizes that code as a homological surface code. For an example of the phaseerror decoder graph of a compass code, see Fig. 4.

The task of a decoder is then, given some syndrome information in the form of marked nodes, to identify the corresponding edge configuration producing those marked nodes up to homology. The question we consider in this paper is the following: What threshold gains do we obtain by modifying the phase and bit-flip decoder graphs according to asymmetrically distributed edge failure probabilities?

\section{Maximum-likelihood decoding}

The maximum-likelihood decoder is one that takes in syndrome information and chooses the most likely error class producing that syndrome. Formally, its probability of success is given by $p_{\text {succ }}=\sum_{s} \operatorname{Pr}\left(\bar{E}_{s}\right)$, where the sum runs over all syndromes $s$ and $\bar{E}_{s}$ is the most likely error class conditioned on syndrome $s$. This decoder will yield optimal thresholds, but it is often inefficient to implement.

Fortunately, one can relate the threshold of the maximum-likelihood decoder to the phase transition of an associated random-bond Ising model [6,40,41], with coupling strengths satisfying a particular set of criteria known as the Nishimori conditions [42]. The probability of an error class is then given by the partition function at a particular quenched disorder. In particular, the disorder of 
the model serves as an order parameter that captures this phase transition, which determines the maximum-likelihood threshold in the presence of independent [41] and even correlated [42] noise. For our purposes, however, we only need the correspondence to independently decoded noise [6].

\section{Minimum-weight perfect matching decoding}

The minimum-weight perfect matching (MWPM) decoder assigns to each syndrome the error class corresponding to a most-likely individual error producing that syndrome. Its probability of success is then $p_{\text {succ }}=\sum_{s} \operatorname{Pr}\left(\overline{E_{s}}\right)$, where $E_{s}$ is the most-likely error producing syndrome $s$.

This decoder is implemented by constructing a minimumweight perfect matching among the marked vertices in the decoder graph. The edge weights between two marked vertices correspond to the most probable path between them, and our edge weights are generated using standard techniques [43]. For symmetric noise, this is simply the shortest path, but for asymmetric noise it need not be. Fortunately, Edmond's blossom algorithm runs efficiently on graphs without hyperedges, taking $O\left(n^{3}\right)$ time on a graph with $n$ nodes [44].

Within the subfamily of compass codes we focus on, each qubit participates in at most two stabilizer generators of a given type. As a result, the corresponding decoder graphs contain no hyperedges, so compass codes inherit the efficient MWPM decoder of the surface code.

When dealing with boundary conditions, some care must be taken to ensure a perfect matching exists since the parity of the marked nodes may no longer be even. We use the techniques of Ref. [14] to estimate the logical error rates in the presence of boundaries.

\section{Asymmetrically weighted union-find decoding}

The final decoder we use is an asymmetrically weighted variant of the union-find decoder recently proposed in Ref. [34]. This decoder is guaranteed to perform optimally on errors of weight at most $\lfloor d / 2\rfloor$, and it has been shown empirically to perform almost as well as MWPM on toric codes with respect to its threshold.

For simplicity, our simulations are run with a periodic north-south boundary condition, which suffices for threshold comparison [45]. However, for completeness, we summarize the decoder on lattices with boundaries, along with our modifications to account for asymmetric error rates. Decoding proceeds in two steps.

(1) Asymmetrically weighted syndrome validation. The first step is (weighted) syndrome validation. In this step, we form an erasure that is consistent with the observed syndrome and that accounts for the asymmetric error rates. To satisfy the first property, we save each node as a cluster, growing all clusters with an odd number of marked nodes by half-edges. After each growth, we fuse those clusters that intersect.
The cluster growth terminates when each cluster has an even number of marked nodes, indicating that we can form a hypothetical erasure that is consistent with the observed syndrome. Furthermore, we use the weighted-growth heuristic, growing only those odd clusters in each step whose boundary is smallest. We refer to the reader to Ref. [34] for a more lengthy description of syndrome validation.

In the case of a decoder graph with a boundary, we no longer have a guarantee that there are an even number of syndromes in our graph. This is because some of the syndromes might condense at the boundary. To accommodate for this issue, we simply treat the boundary as a sink in which every cluster that fuses with the boundary is assigned an even parity.

After this, syndrome verification concludes by choosing a spanning forest within the clusters. We asymmetrically weight syndrome verification by using Kruskal's algorithm to choose a maximumweight spanning forest, where each edge is weighted according to its probability of failure [46]. This choice increases the probability of identifying the erroneous qubits.

(2) Peeling with boundaries. Having associated to the graph an erasure forest that is consistent with the observed syndrome and asymmetric error rates, the second step is to apply maximum-likelihood erasure decoding in the form of an altered peeling decoder [47].

To each leaf node of the resulting erasure forest, we apply the following rules:

(i) If the leaf node is marked, apply a phase flip to the corresponding edge and flip the mark of the connected node. Then, remove the leaf node and edge from the erasure tree.

(ii) If the leaf node is unmarked, remove it and the corresponding edge from the erasure tree.

At this stage, we have an erasure forest with no leaf nodes and potentially some open edges connecting to the open boundaries. Unfortunately, these open edges are missing their leaf nodes, so we cannot peel them. In Ref. [47], this case is avoided by growing the spanning forest so that each tree has at most one open edge, and then peeling towards that edge. However, for asymmetrically distributed noise, a maximumweight spanning forest might not take this form.

Instead, we can use dynamic programming to find a maximum-probability error configuration consistent with syndrome information in linear time. Fix any tree inside the forest, with edges weighted according to their error probabilities, and root the tree at any node. Each node in this tree corresponds to a stabilizer, which will be either marked or unmarked. Our aim is to identify a subset $S$ of edges that is both consistent with the syndrome information 


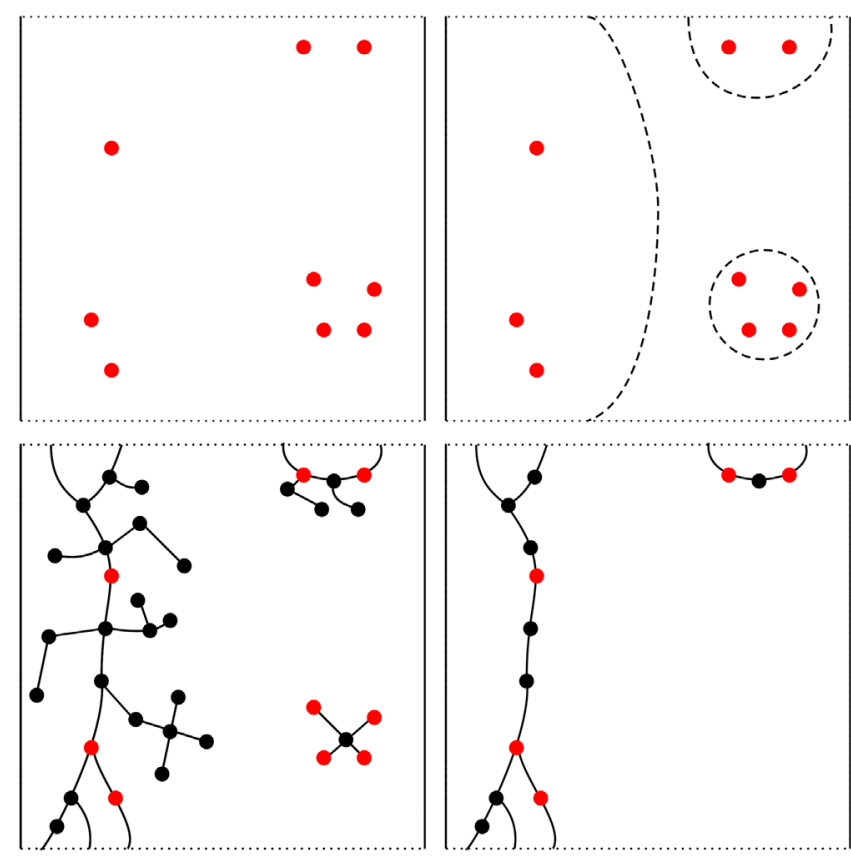

FIG. 2. The square represents the decoder graph (unseen) with north-south open boundary conditions. First, the clusters (dashed enclosures) are grown to an erasure consistent with the (red) marked syndromes. We then find maximum-weight spanning trees, with unmarked syndromes in black. After peeling the leaf nodes, we decode the trees using dynamic programming.

and has maximal failure probability. We then apply our phase-error correction to this set.

We proceed recursively. To each node $v$, we associate two values. First, we compute the maximum weight of $S_{v}$ for the subtree rooted at $v$ over all subspanning trees that include the parent edge. Second, we compute the same maximum weight of $S_{v}$ over all subspanning trees that do not include the parent edge. Each of these updates takes constant time, assuming that the children were previously evaluated and that $v$ has a bounded degree. Iterating over all vertices in the tree and trees within the forest, this terminates in linear time and can be used to produce the desired $S$.

By using a tree structure, the union-find growth algorithm takes $O(n \cdot \alpha(n))$ time, where $\alpha$ is the exceptionally slow-growing inverse Ackermann's function. However, because we find a maximum-weight spanning tree, this variant requires $O(n \log (n))$-time preprocessing. The union-find decoder is the most time efficient of the three decoders we consider.

For a pictoral skeleton of the decoder, see Fig. 2. A comparison of the decoder error rates with and without the asymmetric alteration on the surface code is shown in Fig. 3. There, the error model is generated by choosing an error probability $p_{i} \in_{r}[0,2 p]$ for each physical qubit $i$ uniformly at random. The value $p_{i}$ is passed to the asymmetric decoder to inform Kruskal's algorithm. This

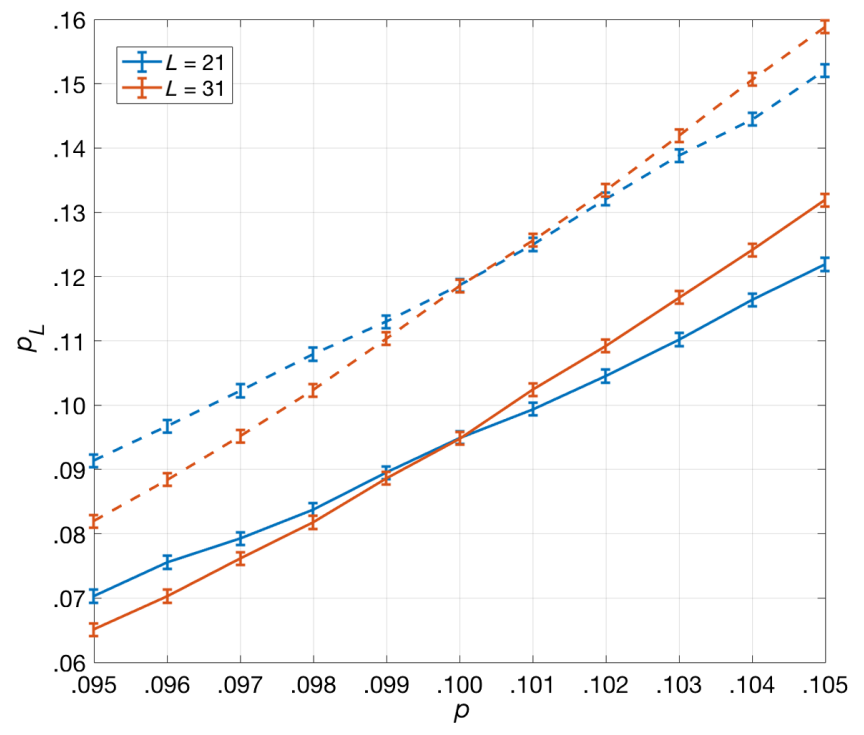

FIG. 3. A comparison of logical error rates for the asymmetric decoder (solid lines) versus the symmetric decoder (dashed lines). Each data point was generated with $10^{6}$ independent Monte Carlo trials.

additional information results in an improvement on the error rate but with little effect on the threshold.

\section{THRESHOLD SCALING}

Before we consider asymmetric noise models, we ask the more fundamental question, how does the threshold behave in these compass codes? In particular, Bacon-Shor codes have no threshold, while surface codes boast some of the highest thresholds. Compass codes provide a framework for interpolating between these two, so we examine the threshold scaling here first.

We use the code's CSS structure to argue directly about phase-flip errors of probability $p$; bit-flip errors can be decoded analogously and independently. To correct phase errors, the relevant information about the code consists of $\mathcal{G}_{Z}$ and $\mathcal{S}_{X}$, the $Z$-type gauge subgroup and the $X$-type stabilizer subgroup, respectively.

\section{A. Surface-density codes}

The first family of codes we consider are the (randomized) surface-density codes, which interpolate between the Bacon-Shor and surface codes. Each code is determined stochastically according to a surface-density $q_{\text {surf }}$ in the following way. Given a square lattice, for each plaquette of one color in the checkerboard configuration of the surface code, we cut the corresponding $X$-type stabilizer at that plaquette with probability $q_{\text {surf }}$. Correspondingly, $q_{\text {surf }}=0$ is equivalent to the Bacon-Shor family (with respect to phase errors), and $q_{\text {surf }}=1$ is equivalent to the surface code. 


\section{Ising models associated to quantum codes}

We identify the scaling of the threshold with the surfacedensity under maximum-likelihood decoding. To do so, we exploit the aforementioned correspondence between thresholds of quantum codes and phase transitions of associated random-bond Ising models [6,40-42].

We briefly summarize this connection in the simplest case of independently decoded noise. Let $\mathcal{G}_{0}$ be a minimal generating set of $\mathcal{G}_{Z}$. Let the $g_{i} \in \mathcal{G}_{0}$ be indexed by $i$, and associate to each generator an Ising spin $s_{i}= \pm 1$. Index the physical qubits by $j \in\left\{1, \ldots, L^{2}\right\}$, and define

$$
g_{i}(j):= \begin{cases}1 & \text { if } g_{i} \text { is supported on site } j \\ 0 & \text { otherwise }\end{cases}
$$

Then, for any vector $\tau \in\{+1,-1\}^{L^{2}}$, we define the classical spin Hamiltonian

$$
H_{\tau}(s)=-\sum_{j=1}^{L^{2}} \tau_{j} \prod_{i=1}^{\left|\mathcal{G}_{0}\right|} s_{i}^{g_{i}(j)} .
$$

For any Pauli $Z$ error $E$, define $\left(\tau_{E}\right)_{k}$ to be -1 if $E$ is supported on site $k$, and +1 otherwise. For physical error rate $p$, we can define the virtual temperature $\beta_{p}$ according to the Nishimori line [48] so that

$$
\beta_{p}:=\frac{\log (1-p)-\log (p)}{2} .
$$

Note that this is a simplification of the more general Nishimori conditions, with fixed coupling coefficients $J=1$ [42]. Define $\tau$ to be a quenched vectorvalued random variable that takes value $\tau_{E}$ with probability $p^{|E|}(1-p)^{L^{2}-|E|}$. Then, a phase transition of the associated random-bond Ising model at $\left(p_{c}, \beta_{p_{c}}\right)$ corresponds to an accuracy threshold at $p_{c}[6,40-42]$.

For an example of an Ising model associated to a compass code, see Fig. 4. Note that, for decoder graphs without hyperedges, the graph defining the Ising model is dual to the decoder graph.

\section{Numerical simulations}

Parameters of the simulation.-We map surface-density codes to their corresponding anisotropic Ising models on random graphs. We generate random samples of the model with the given $q_{\text {surf }}$ and $p$ for various system sizes $L$, with the temperature determined by the Nishimori line according to the disorder parameter $p$. For each random trial, we use a cluster algorithm [49] and an improved estimator to compute the Binder cumulant [50]. Finally, we scan over $p$ [at a separation of 0.1 for $\ln (p)]$ and look for a transition point. The system size we use ranges from $L=5$ to $L=61$, the number of steps for the cluster update ranges
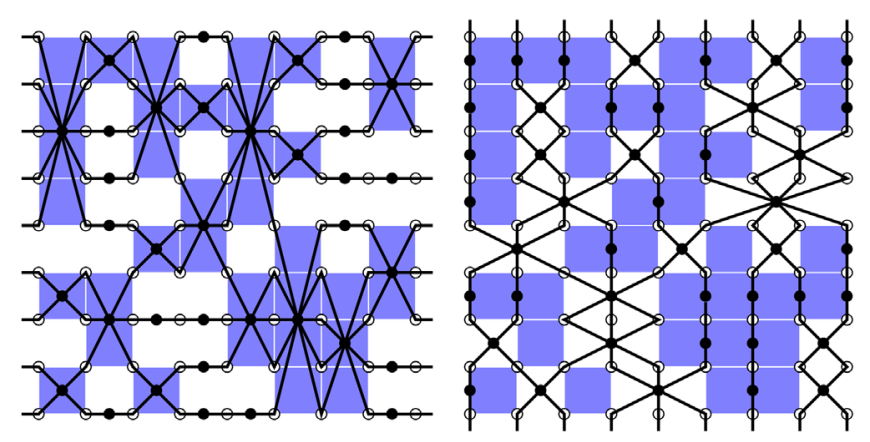

FIG. 4. The left-hand side represents the graph describing the two-body Ising model. The right-hand side represents its dual, the decoder graph. The blue squares represent cuts in the $X$-type stabilizers on a $9 \times 9$ lattice. The connectivity on the left-hand graph determines the sparsity on the right.

from $10^{6}$ to $10^{8}$, and the number of random trials for each parameter set ranges from 200 to $10^{4}$.

In general, as the transition point $p_{c}$ increases with $q_{\text {surf }}$, it enhances the frustration in the system, so more steps are needed for convergence. This case is verified by the autocorrelation of the observables. However, for larger $q_{\text {surf }}$, the slope of the Binder cumulant $U$ with respect to $-\ln (p)$ also increases. As a result, less samples and smaller system sizes are required to achieve the same level of accuracy.

Numerical results.-Interestingly, simulations suggest that the threshold grows linearly with the surface density (see Fig. 5). In particular, a positive density is both

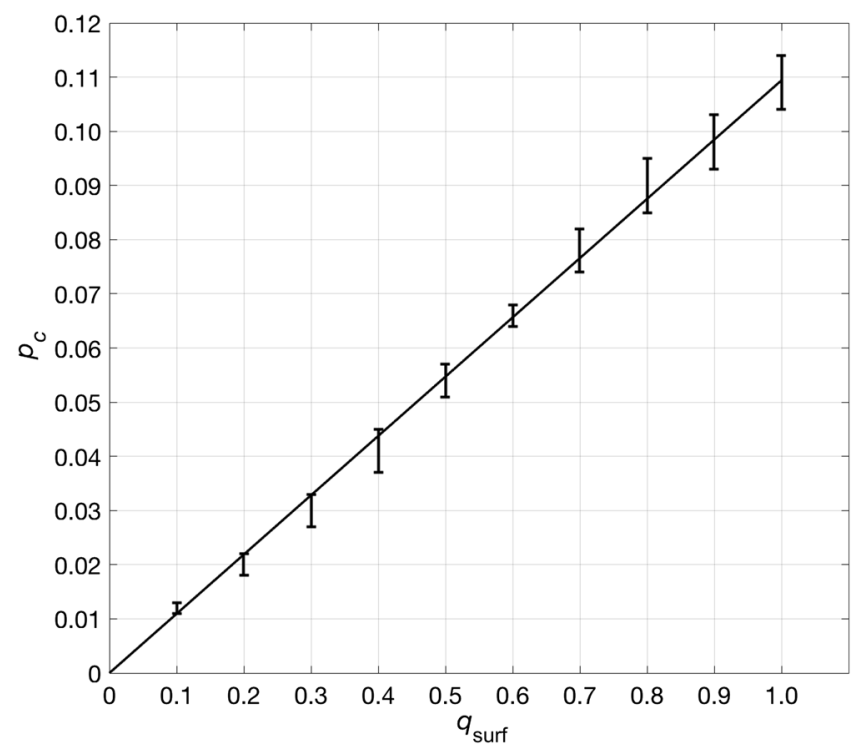

FIG. 5. Scaling of the critical disorder $p_{c}$ with respect to the surface density $q_{\text {surf }}$. Autocorrelation is checked using a binning analysis; the fit is linear through the origin. The widest error bars are of total width of approximately $1 \%$. At $q_{\text {surf }}=1$, the results closely match the established critical point at $p_{c}=0.1094 \pm$ 0.0002 [51]. 
necessary and sufficient for the presence of a threshold. The linearity contrasts with the threshold scaling of the less-restricted code family that we consider next.

\section{B. Shor-density codes}

We next turn our attention to Shor-density codes, which form a randomized family of codes that interpolate between Bacon-Shor codes and their full $X$-type gauge-fix, Shor's subspace code. These codes are defined similarly to surface-density codes according to a new parameter, which we call the Shor-density $q_{\text {shor }}$ For these codes, $X$-type stabilizers are cut at each plaquette with probability $q_{\text {shor }}$. Thus, $q_{\text {shor }}=0$ again corresponds to the Bacon-Shor code, whereas $q_{\text {shor }}=1$ corresponds to Shor's subspace code [52].

Of course, the thresholds for such codes are one-sided: More cuts for one type of stabilizer leaves less for the other. Consequently, such codes are best suited for asymmetric noise models. Note that these codes remain local, in the sense that the expected maximum stabilizer weight grows logarithmically in the lattice size for any fixed $q_{\text {shor }}$.

Because the associated graphs to these codes have a richer structure, which may hinder the convergence of the clustering algorithm, we instead study these codes using the union-find decoder. We generate a new decoder graph and error in each round and perform $10^{6}$ Monte Carlo trials for each data point. We then exploit the efficiency of the unionfind decoder to run 300 Monte Carlo trials on a $1001 \times$ 1001 lattice to verify the thresholds, which should sharply converge to either $p_{L}=0$ or $p_{L}=0.5$ about the threshold. This large lattice size is necessary to mitigate the growing finite-size effects.

The threshold scaling in Fig. 6 nearly saturates the zerorate quantum Gilbert-Varshamov bound [38],

$$
H\left(p_{x}\right)+H\left(p_{z}\right) \leq 1,
$$

mirroring results obtained on other lattice configurations $[53,54]$. One thing to note is the normalizing finite-size effects at very high and very low densities. Note that, at $q_{\text {shor }}=1$, we essentially have disjoint copies of a repetition code. This result has a threshold of 50\% since the unionfind decoder behaves optimally on errors of weight less than $\lfloor L / 2\rfloor[34]$. However, we observe a pseudothreshold of approximately $45 \%$ for union-find decoding on a $1001 \times$ 1001 lattice, matching the analytical solution

$$
p_{\text {logical }}=\frac{1}{2}\left(1-\left(1-2 p_{\text {rep }}\right)^{L}\right)
$$

where $p_{\text {rep }}$ is the probability of failure of a repetition code of length $L$,

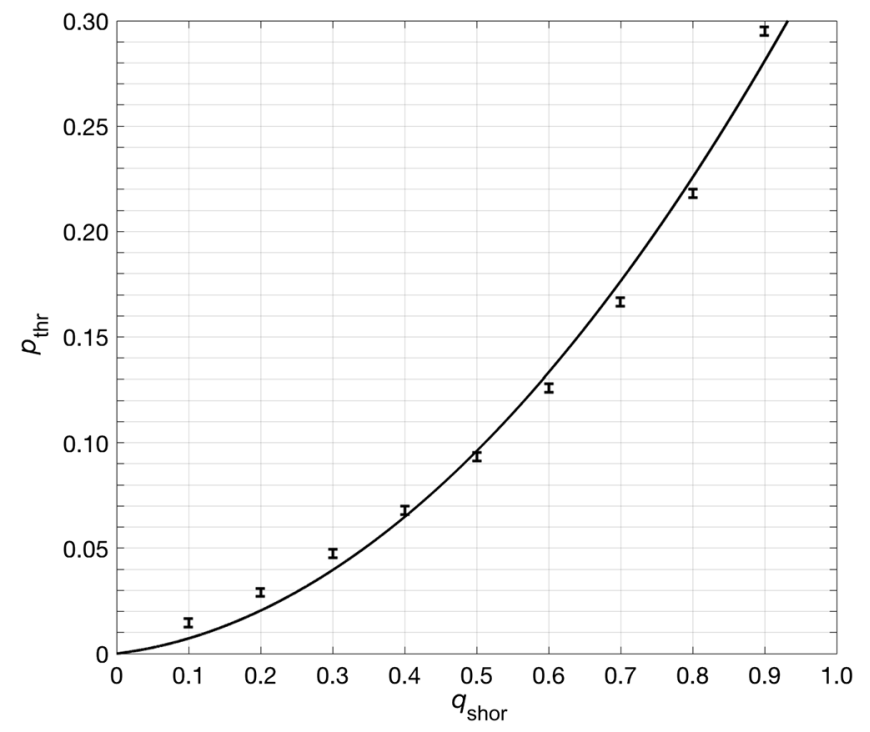

FIG. 6. Scaling of the estimated threshold $p$ with respect to the Shor density $q_{\text {shor }}$. The fit is quadratic through the origin; the finite-size effects are apparent. All points were obtained on $81 \times 81$-size lattices, except for $q_{\text {shor }}=0.9$. To emphasize finitesize effects, this was performed on a $631 \times 631$ lattice, which is greater than necessary for fault-tolerant computation [10].

$$
p_{\text {rep }}=\sum_{k=\left\lceil\frac{L}{2}\right\rceil}^{L}\left(\begin{array}{l}
L \\
k
\end{array}\right) p^{k}(1-p)^{L-k}
$$

Summary.-These simulations suggest that the threshold is determined predominantly by the density of syndrome measurements, rather than their specific configuration, for symmetrically distributed noise. The usual surface code does not far outperform randomized codes of equal density by this metric; it does so only slightly, as its symmetry will minimize the number of $\lceil L / 2\rceil$-weight errors that introduce a logical error. This result is reinforced by the observation that the threshold appears to scale linearly with surface density, but it is strictly convex with respect to Shor density.

\section{ASYMMETRIC NOISE}

Next, we turn our attention to asymmetric noise. We consider two different types: biased noise that is symmetrically distributed throughout the lattice, and asymmetrically distributed noise. In both cases, we find that substantial gains can be made by tailoring the decoder graphs to the noise directly. We analyze these in both the code capacity and phenomenological models, and compute their thresholds under different noise biases.

\section{A. Biased but symmetric noise}

For biased noise that is symmetrically distributed, we construct a family of compass codes we call elongated codes. These codes are defined by a parameter $\ell \in \mathbb{N}^{+}$that 
we call the elongation of the code, and they are constructed by cutting the $Z$ stabilizers at the $(i, j)$ th plaquettes for all $i-j \equiv 0(\bmod \ell)$. The $X$ stabilizers are then cut at all remaining plaquettes, resulting in a subspace code. This method is similar to the approaches of Refs. [16,24], which consider concatenations with different phase-flip repetition codes.

Under this definition, we obtain Shor's code for $\ell=1$ and the surface code for $\ell=2$. For $\ell>2$, we obtain an asymmetrization of Kitaev's toric code in the bulk with extended $2 \ell$-body plaquette operators. This family illustrates that a simple compass code is well equipped to correct asymmetric noise while somewhat sacrificing locality.

It is worth noting that choosing asymmetric lattice dimensions as in Ref. [4] may alter the logical error rate of a code family, but it will not change the threshold, as it is a property of the bulk. Thus, the elongation of the code refers to a stretching of the bulk stabilizer geometry not the lattice itself.

As the elongation grows, finite-size effects play a greater role. As such, we use MWPM decoding to perform simulations on smaller lattices at lower elongations and union-find decoding to test larger lattices. While these larger lattices also suffer from finite-size effects, we use the efficiency of union-find decoding to simulate lattices of between $10^{3}$ and $10^{4}$ qubits, which is the estimated code size required for full-scale fault-tolerant computation [10].

Furthermore, we estimate the phenomenological threshold by simulating $(2+1)-D$ elongated codes. For a physical lattice of linear size $L$, this corresponds to performing $L$ rounds of faulty syndrome extraction, followed by an ideal round, and then decoding. The corresponding decoder graph is then $L+1$ copies of the initial decoder graph, with $L$ timelike slices of edges connecting the corresponding vertices in each spacelike slice. These timelike edges represent faulty measurements.

Although the size of each stabilizer is independent of the lattice size, we scale the probability of failure for each stabilizer linearly with its weight. We assume the usual phenomenological normalization that plaquette stabilizers are faulty at the physical error rate $p$. Despite some increasing stabilizer weights, we observe substantial threshold gains in both the code capacity and phenomenological models.

Tables I and II show the code-capacity thresholds using the MWPM and union-find decoder, respectively, while Table III shows the phenomenological threshold using the union-find decoder. In these tables, $\eta_{\text {opt }}$ refers to the optimal bias that realizes the threshold $p_{\text {thr }}$, while $\eta_{*}$ is the bias above which the codes will outperform the surface code.

Notably, a relatively smaller noise bias is required to outperform the surface code in the phenomenological setting. Unsurprisingly, the MWPM outperforms the union-find decoder as a whole, but surprisingly, it displays
TABLE I. Thresholds for the MWPM decoder in the codecapacity model. Simulations were done on lattices of size $17 \times 17$ at most.

\begin{tabular}{clcccr}
\hline \hline$\ell$ & $\eta_{\mathrm{opt}}$ & $p_{\mathrm{thr}}$ & $\eta_{*}$ & $p_{z}$ & \multicolumn{1}{c}{$p_{x}$} \\
\hline 2 & 0.5 & $15.5 \%$ & $N / A$ & $10.3 \% \pm 0.2 \%$ & $10.3 \% \pm 0.2 \%$ \\
3 & 1.67 & $17.9 \%$ & 1.39 & $14.1 \% \pm 0.3 \%$ & $6.5 \% \pm 0.2 \%$ \\
4 & 3.00 & $20.0 \%$ & 2.10 & $17.5 \% \pm 0.2 \%$ & $5.0 \% \pm 0.2 \%$ \\
5 & 4.26 & $21.6 \%$ & 2.78 & $19.5 \% \pm 0.1 \%$ & $4.1 \% \pm 0.1 \%$ \\
6 & 5.89 & $22.8 \%$ & 3.70 & $21.1 \% \pm 0.1 \%$ & $3.3 \% \pm 0.1 \%$ \\
\hline \hline
\end{tabular}

TABLE II. Thresholds for the union-find decoder in the codecapacity model. Simulations were done on lattices of size $81 \times 81$ at most.

\begin{tabular}{lccccr}
\hline \hline$\ell$ & $\eta_{\mathrm{opt}}$ & $p_{\text {thr }}$ & $\eta_{*}$ & $p_{z}$ & $p_{x}$ \\
\hline 2 & 0.5 & $15.0 \%$ & $N / A$ & $10.0 \% \pm 0.2 \%$ & $10.0 \% \pm 0.2 \%$ \\
3 & 1.41 & $16.9 \%$ & 1.14 & $13.4 \% \pm 0.3 \%$ & $7.0 \% \pm 0.2 \%$ \\
4 & 2.40 & $18.4 \%$ & 1.78 & $15.7 \% \pm 0.2 \%$ & $5.4 \% \pm 0.2 \%$ \\
5 & 3.45 & $19.6 \%$ & 2.41 & $17.4 \% \pm 0.1 \%$ & $4.4 \% \pm 0.1 \%$ \\
6 & 4.45 & $20.7 \%$ & 2.95 & $18.8 \% \pm 0.1 \%$ & $3.8 \% \pm 0.1 \%$ \\
7 & 5.62 & $21.9 \%$ & 3.55 & $20.2 \% \pm 0.1 \%$ & $3.3 \% \pm 0.1 \%$ \\
8 & 6.23 & $22.8 \%$ & 3.84 & $21.2 \% \pm 0.1 \%$ & $3.1 \% \pm 0.1 \%$ \\
9 & 7.29 & $23.7 \%$ & 4.17 & $22.2 \% \pm 0.1 \%$ & $2.9 \% \pm 0.1 \%$ \\
10 & 8.36 & $24.0 \%$ & 4.77 & $22.7 \% \pm 0.1 \%$ & $2.6 \% \pm 0.1 \%$ \\
20 & 20.7 & $28.3 \%$ & 10.5 & $27.6 \% \pm 0.1 \%$ & $1.3 \% \pm 0.1 \%$ \\
50 & 55.3 & $33.8 \%$ & 24.0 & $33.5 \% \pm 0.1 \%$ & $0.6 \% \pm 0.1 \%$ \\
\hline \hline
\end{tabular}

TABLE III. Thresholds for the union-find decoder in the phenomenological model. Simulations were done on lattices of size $35 \times 35 \times 35$ at most.

\begin{tabular}{lllcrr}
\hline \hline$\ell$ & $\eta_{\mathrm{opt}}$ & $p_{\mathrm{thr}}$ & $\eta_{*}$ & $p_{z}$ & \multicolumn{1}{c}{$p_{x}$} \\
\hline 2 & 0.5 & $3.98 \%$ & $N / A$ & $2.65 \% \pm 0.2 \%$ & $2.65 \% \pm 0.2 \%$ \\
3 & 1.20 & $4.45 \%$ & 0.99 & $3.4 \% \pm 0.2 \%$ & $2.0 \% \pm 0.2 \%$ \\
4 & 1.88 & $4.60 \%$ & 1.49 & $3.8 \% \pm 0.2 \%$ & $1.6 \% \pm 0.2 \%$ \\
5 & 2.73 & $4.85 \%$ & 2.06 & $4.2 \% \pm 0.2 \%$ & $1.3 \% \pm 0.2 \%$ \\
6 & 3.17 & $5.00 \%$ & 2.32 & $4.4 \% \pm 0.2 \%$ & $1.2 \% \pm 0.2 \%$ \\
\hline \hline
\end{tabular}

lower thresholds on lattices comprised of higher-weight stabilizers. This result suggests that union-find decoding may better exploit the degeneracy of certain lattices; in particular, one should use MWPM for Z-type errors and union-find decoding for $X$-type errors on elongated lattices. Our estimates for established surface-code thresholds match those found in Ref. [14] at $10.3 \%$ for MWPM decoding and in Ref. [34] at $9.95 \%$ and $2.65 \%$ for unionfind decoding in 2 - and $(2+1)-D$, respectively.

\section{B. Spatially dependent noise}

We conclude by considering noise that is asymmetrically distributed throughout the lattice. To illustrate the idea, we 

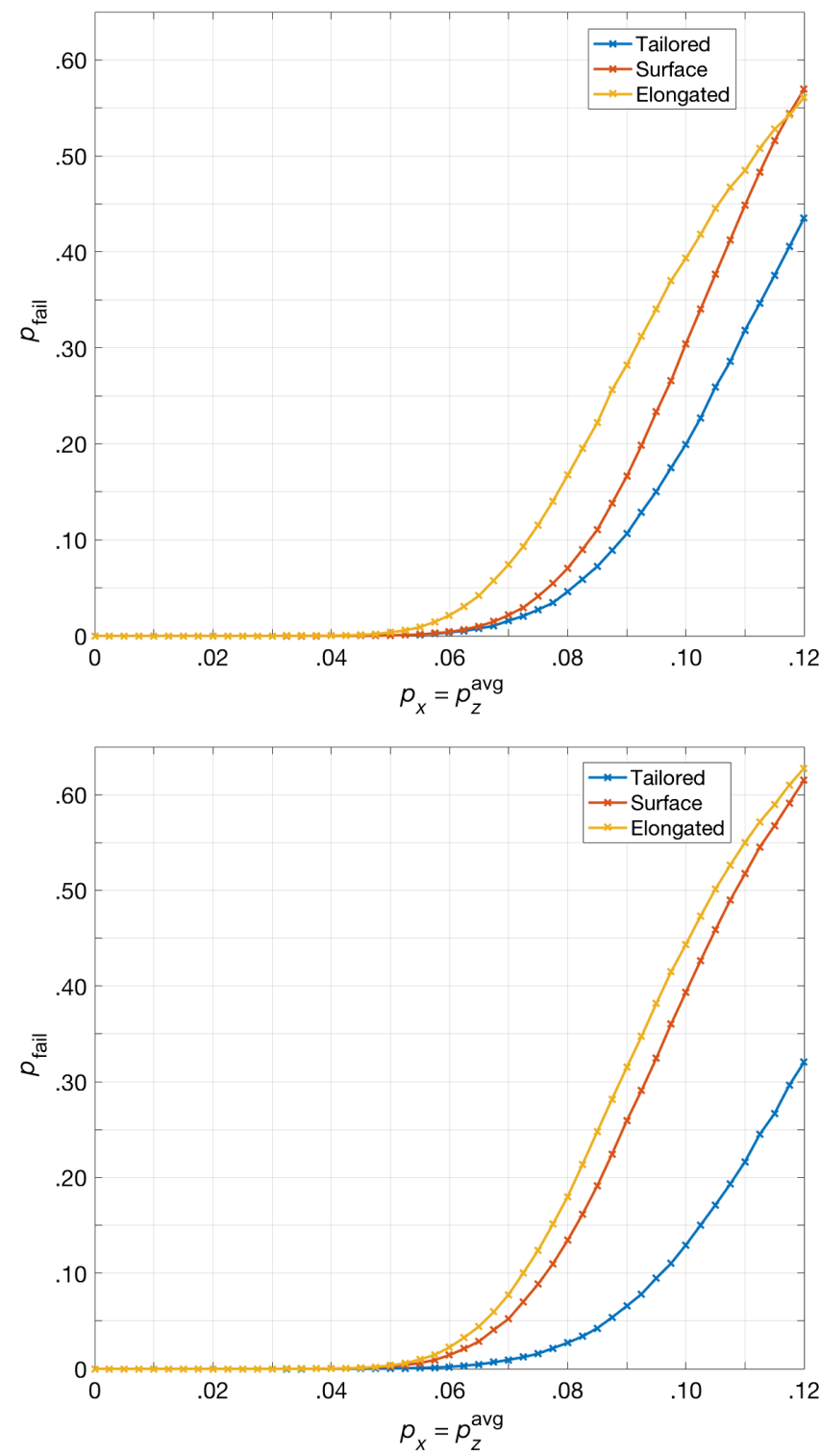

FIG. 7. Error rates for gradually ( $w=0.25$, top panel) and steeply ( $w=0.10$, bottom panel) inclined linear noise, computed on a lattice of size $33 \times 33$ using union-find decoding in the highnoise regime. Here, $p_{\text {fail }}$ represents the total probability of a failure in either the $X$ - or $Z$-type decoders.

focus on a simple noise model in which dephasing noise decays linearly from the right-hand side of the lattice according to the function $p_{z}(i, j)=(w(j / L)+(1-w) \times$ $(1-j / L)) p_{\text {tot }} / 2$. Here, $i$ and $j$ are the coordinates of a qubit, $L$ is the linear size of the lattice, and $w$ is a constant that determines the degree of incline. We further assume that $p_{x}=p_{\text {tot }} / 2$, so $p_{\text {tot }}$ is the total infidelity of the channel. Note that the average bias between the dephasing noise and bit-flip noise is symmetric.

The idea is simple: When the noise is distributed asymmetrically, the stabilizer information can be chosen to match the noise. Intuitively, lower weight stabilizers add more error information about the qubits nearby. With this in mind, we define a randomized family of codes that we call $\left(p_{z}-\right)$ tailored codes. At each plaquette, we choose to cut the corresponding $X$-type stabilizer with probability $2 p_{z}(i, j) / p_{\text {tot }}$, where $i, j$ are the coordinates of the upperleft qubit at that plaquette. Then, in the presence of a high amount of dephasing noise, many low-weight $X$-type stabilizers will appear to aide in error correction.

We observe that the tailoring of these codes to the noise model can augment error rates (see Fig. 7). It is worth noting, however, that simply weighting the probability of each cut according to the surrounding qubits may not always be the optimal strategy. In particular, in the low error-rate limit, this will become an optimization problem that seeks to minimize the weights of uncorrectable paths of length $\lceil L / 2\rceil$ in the decoder graph.

\section{FAULT TOLERANCE WITH BARE ANCILLA}

One of the major advantages that comes with the locality of the Bacon-Shor code is fault-tolerant bare-ancilla syndrome extraction [16,17]. Although this extraction scheme is the simplest and least resource intensive, most codes incur some loss in effective distance due to high-weight correlated errors produced by errors on the ancilla. For the standard and rotated surface codes, these "hook" errors can be carefully designed to ensure no significant loss in performance $[6,8]$.

In the compass-code framework, this resilience to correlated errors is a general phenomenon resulting from measuring stabilizers along the Bacon-Shor gauge operators. Using such a syndrome extraction scheme on any gauge fix of the Bacon-Shor code, any collection of $d-1$ faults in the circuit produce an error of the form $E G$, where $|\operatorname{supp}(E)| \leq d-1$ is minimal and $G$ is a gauge operator of the initial Bacon-Shor code.

Divide the generators of the stabilizer group of any compass code into $\mathcal{S}=\left\langle\mathcal{S}_{B}, \mathcal{S}_{F}\right\rangle$, where $\mathcal{S}_{B}$ are the stabilizer generators of the Bacon-Shor code and $\mathcal{S}_{F}$ are those gauge operators that have been fixed. Then, for any error $E G$ resulting from $d-1$ faults in the circuit, if $|\operatorname{supp}(E)|=0$, then either $G \in \mathcal{S}_{F}$ or there exists an $S \in \mathcal{S}_{F}: S G=-G S$. Otherwise, if $0<|\operatorname{supp}(E)|<d$, then there exists an $S \in \mathcal{S}_{B}: S E=-E S$. Since $S$ must also commute with any gauge operator $G$, it follows that $E G$ is detectable. Thus, any error resulting from $\leq d-1$ faults during syndrome extraction is either detectable or trivial.

This demonstrates that there exists fault-tolerant decoding without a loss in effective distance. However, it is not necessarily maximum-likelihood decoding on the memory. One simple counterexample is Shor's code, where a single well-placed ancilla error can affect a weight $d$ memory error that maximum-likelihood will misdiagnose as a weight $d-1$ memory error, resulting in failure. The above case implies that performing MWPM with respect to linearprobability faults in the decoder graph is fault tolerant. Introducing these faults amounts to triangulating the 
decoding graph, similar to hook errors in the surface-code case $[6,14]$. Determining circuit-level compass-code performance in this model is the subject of future inquiry [55].

\section{CONCLUSIONS}

In this article, we have described an ansatz for designing planar codes stemming from the 2D compass model. We have provided evidence that simple subfamilies of this class may be useful for correcting biased noise in idealized code capacity and phenomenological noise models, particularly if that bias is distributed geometrically. In particular, one can bias the stabilizers locally towards correcting a certain error type.

There are two central challenges for these codes in the more realistic circuit-level noise model. Although these codes are still local, there is a trade-off between the bias of the codes and the locality of the stabilizer measurements. We have demonstrated that fault-tolerant measurement in Bacon-Shor [16,17] and surface codes [6,8] using bare ancilla can be adapted to the compass model, if measurements are performed in the correct order. Nevertheless, these correlated errors will deteriorate code performance as higher-weight stabilizer outcomes become less reliable. This result might be mitigated by using other flag-type schemes or by preserving some gauge d.o.f. We would expect that these gains would persist but at the expense of higher bias and code overhead. As such, we leave a more involved circuit-level analysis to future work.

The second concern is whether the biased noise model itself can persist at the circuit level. To remain experimentally motivated, one must choose operations that preserve the bias [5,22,24]. Consequently, the construction of simple and bias-preserving fault-tolerant gadgets is key to utilizing asymmetric noise.

Finally, we have only narrowly broached the design space offered by these codes. Exploring different configurations according to other geometrically defined noise [32], generalizing to codes defined on the 3-D compass model, and using correlated decoders $[5,25-27,56]$ are all avenues to explore. More generally, finding other low-density parity-check constructions adapted to biased noise may give the best of both worlds, mitigating the overhead of asymmetrization while taking advantage of the bias.

\section{ACKNOWLEDGMENTS}

The authors thank Dave Bacon, Nicolas Delfosse, and Pavithran Iyer for useful discussions, and Luming Duan for providing computational resources for simulations on the Flux Cluster at the University of Michigan. Additionally, they thank anonymous referees for their helpful comments. This research was supported in part by National Science Foundation (NSF) Grant No. 1717523, the ODNI/IARPA LogiQ Program (Grant No. W911NF-10-1-0231), ARO MURI (Grant No. W911NF-16-1-0349), EPiQC-An NSF
Expedition in Computing (Grant No. 1730104), and the Alexander von Humboldt Foundation.

[1] P. Aliferis, D. Gottesman, and J. Preskill, Quantum Accuracy Threshold for Concatenated Distance-3 Codes, Quantum Inf. Comput. 6, 97 (2005).

[2] E. Knill, R. Laflamme, and W. Zurek, Threshold Accuracy for Quantum Computation, arXiv:quant-ph/9610011.

[3] D. Aharonov and M. Ben-Or, Fault-Tolerant Quantum Computation with Constant Error, in Proceedings of the 29th Annual ACM Symposium on Theory of Computing (ACM, New York, 1997), pp. 176-188.

[4] J. Napp and J. Preskill, Optimal Bacon-Shor Codes, Quantum Inf. Comput. 13, 490 (2013).

[5] D. K. Tuckett, S. D. Bartlett, and S. T. Flammia, Ultrahigh Error Threshold for Surface Codes with Biased Noise, Phys. Rev. Lett. 120, 050505 (2018).

[6] E. Dennis, A. Kitaev, A. Landahl, and J. Preskill, Topological Quantum Memory, J. Math. Phys. (N.Y.) 43, 4452 (2002).

[7] H. Bombín, Gauge Color Codes: Optimal Transversal Gates and Gauge Fixing in Topological Stabilizer Codes, New J. Phys. 17, 083002 (2015).

[8] Yu Tomita and K. M. Svore, Low-Distance Surface Codes under Realistic Quantum Noise, Phys. Rev. A 90, 062320 (2014).

[9] T. J. Yoder and I. H. Kim, The Surface Code with a Twist, Quantum 1, 2 (2017).

[10] A. G. Fowler, M. Mariantoni, J. M. Martinis, and A. N. Cleland, Surface Codes: Towards Practical Large-Scale Quantum Computation, Phys. Rev. A 86, 032324 (2012).

[11] B. M. Terhal, Quantum Error Correction for Quantum Memories, Rev. Mod. Phys. 87, 307 (2015).

[12] T. E. Obrien, B. Tarasinski, and L. DiCarlo, Density-Matrix Simulation of Small Surface Codes under Current and Projected Experimental Noise, npj Quantum Inf. 3, 39 (2017).

[13] C. J. Trout, M. Li, M. Gutiérrez, Y. Wu, S.-T. Wang, L. Duan, and K. R. Brown, Simulating the Performance of a Distance-3 Surface Code in a Linear Ion Trap, New J. Phys. 20, 043038 (2018).

[14] D. S. Wang, A. G. Fowler, A. M. Stephens, and L. C. L. Hollenberg, Threshold Error Rates for the Toric and Surface Codes, Quantum Inf. Comput. 10, 456 (2010).

[15] D. Bacon, Operator Quantum Error-Correcting Subsystems for Self-Correcting Quantum Memories, Phys. Rev. A 73, 012340 (2006).

[16] P. Aliferis and A. W. Cross, Subsystem Fault Tolerance with the Bacon-Shor Code, Phys. Rev. Lett. 98, 220502 (2007).

[17] M. Li, D. Miller, and K. R. Brown, Direct Measurement of Bacon-Shor Code Stabilizers, Phys. Rev. A 98, 050301 (2018).

[18] T. J. Yoder, Universal Fault-Tolerant Quantum Computation with Bacon-Shor Codes, arXiv:1705.01686.

[19] F. Pastawski, A. Kay, N. Schuch, and I. Cirac, Limitations of Passive Protection of Quantum Information, Quantum Inf. Comput. 10, 580 (2010). 
[20] Z. Nussinov and J. Van Den Brink, Compass Models: Theory and Physical Motivations, Rev. Mod. Phys. 87, 1 (2015).

[21] N. Delfosse, P. Iyer, and D. Poulin, Generalized Surface Codes and Packing of Logical Qubits, arXiv:1606.07116.

[22] P. Aliferis and J. Preskill, Fault-Tolerant Quantum Computation Against Biased Noise, Phys. Rev. A 78, 052331 (2008).

[23] P. Webster, S. D. Bartlett, and D. Poulin, Reducing the Overhead for Quantum Computation When Noise Is Biased, Phys. Rev. A 92, 062309 (2015).

[24] A. M. Stephens, W. J. Munro, and K. Nemoto, HighThreshold Topological Quantum Error Correction Against Biased Noise, Phys. Rev. A 88, 060301(R) (2013).

[25] N. Delfosse and J.-P. Tillich, A Decoding Algorithm for CSS Codes Using the X/Z Correlations, in 2014 IEEE International Symposium on Information Theory (IEEE, New York, 2014), pp. 1071-1075.

[26] N. H. Nickerson and B. J. Brown, Analysing Correlated Noise in the Surface Code Using Adaptive Decoding Algorithms, Quantum 3, 131 (2019).

[27] A.S. Darmawan and D. Poulin, An Efficient General Decoding Algorithm for the Surface Code, Phys. Rev. E 97, 051302 (2018).

[28] A. Kay, Implications of ignorance for quantum-errorcorrection thresholds, Phys. Rev. A 89, 032328 (2014).

[29] A. Robertson, C. Granade, S. D. Bartlett, and S. T. Flammia, Tailored Codes for Small Quantum Memories, Phys. Rev. Applied 8, 064004 (2017).

[30] P. Brooks and J. Preskill, Fault-Tolerant Quantum Computation with Asymmetric Bacon-Shor Codes, Phys. Rev. A 87, 032310 (2013).

[31] P. Aliferis, F. Brito, D. P. DiVincenzo, J. Preskill, M. Steffen, and B. M. Terhal, Fault-Tolerant Computing with Biased-Noise Superconducting Qubits: A Case Study, New J. Phys. 11, 013061 (2009).

[32] N. Delfosse, P. Iyer, and D. Poulin, A Linear-Time Benchmarking Tool for Generalized Surface Codes, arXiv: 1611.04256.

[33] H. Bombín, Topological Subsystem Codes, Phys. Rev. A 81, 032301 (2010).

[34] N. Delfosse and N.H. Nickerson, Almost-Linear Time Decoding Algorithm for Topological Codes, arXiv:1709 .06218 .

[35] J. Dorier, F. Becca, and F. Mila, Quantum Compass Model on the Square Lattice, Phys. Rev. B 72, 024448 (2005).

[36] K. I. Kugel and D. I. Khomskii, Crystal Structure and Magnetic Properties of Substances with Orbital Degeneracy, Zh. Eksp. Teor. Fiz. 64, 1429 (1973).

[37] A. Paetznick and B. W. Reichardt, Universal Fault-Tolerant Quantum Computation with Only Transversal Gates and Error Correction, Phys. Rev. Lett. 111, 090505 (2013).

[38] A. R. Calderbank and P. W. Shor, Good Quantum ErrorCorrecting Codes Exist, Phys. Rev. A 54, 1098 (1996).
[39] A. M. Steane, Error Correcting Codes in Quantum Theory, Phys. Rev. Lett. 77, 793 (1996).

[40] A. Kubica, M. E. Beverland, F. Brandão, J. Preskill, and K. M. Svore, Three-Dimensional Color Code Thresholds via Statistical-Mechanical Mapping, Phys. Rev. Lett. 120, 180501 (2018).

[41] H. Bombin, R. S. Andrist, M. Ohzeki, H. G. Katzgraber, and M. A. Martin-Delgado, Strong Resilience of Topological Codes to Depolarization, Phys. Rev. X 2, 021004 (2012).

[42] C. T. Chubb and S. T. Flammia, Statistical Mechanical Models for Quantum Codes with Correlated Noise, arXiv: 1809.10704.

[43] D. S. Wang, A. G. Fowler, and L. C. L. Hollenberg, Surface Code Quantum Computing with Error Rates Over 1\%, Phys. Rev. A 83, 020302(R) (2011).

[44] J. Edmonds, Paths, Trees, and Flowers, in Classic Papers in Combinatorics (Springer, New York, 2009), pp. 361-379.

[45] A. G. Fowler, Accurate Simulations of Planar Topological Codes Cannot Use Cyclic Boundaries, Phys. Rev. A 87, 062320 (2013).

[46] J. B. Kruskal, On the Shortest Spanning Subtree of a Graph and the Traveling Salesman Problem, Proc. Am. Math. Soc. 7, 48 (1956).

[47] N. Delfosse and G. Zémor, Linear-Time Maximum Likelihood Decoding of Surface Codes Over the Quantum Erasure Channel, arXiv:1703.01517.

[48] H. Nishimori, Geometry-Induced Phase Transition in the $\pm J$ Ising Model, J. Phys. Soc. Jpn. 55, 3305 (1986).

[49] V.S. Dotsenko, W. Selke, and A. L. Talapov, Cluster Monte Carlo Algorithms for Random Ising Models, Physica A 170, 278 (1991).

[50] K. Binder, Finite Size Scaling Analysis of Ising Model Block Distribution Functions, Z. Phys. B 43, 119 (1981).

[51] A. Honecker, M. Picco, and P. Pujol, Universality Class of the Nishimori Point in the $2 \mathrm{D} \pm J$ Random-Bond Ising Model, Phys. Rev. Lett. 87, 047201 (2001).

[52] P. W. Shor, Scheme for Reducing Decoherence in Quantum Computer Memory, Phys. Rev. A 52, R2493 (1995).

[53] K. Fujii and Y. Tokunaga, Error and Loss Tolerances of Surface Codes with General Lattice Structures, Phys. Rev. A 86, 020303(R) (2012).

[54] B. Röthlisberger, J. R. Wootton, R. M. Heath, J. K. Pachos, and D. Loss, Incoherent Dynamics in the Toric Code Subject to Disorder, Phys. Rev. A 85, 022313 (2012).

[55] S. Huang and K. R. Brown (to be published).

[56] N. Maskara, A. Kubica, and T. Jochym-O'Connor, Advantages of Versatile Neural-Network Decoding for Topological Codes, arXiv:1802.08680.

Correction: Errors in a displayed equation in Sec. II A and in an inline equation in the first sentence of the second paragraph of Sec. II A have been fixed. 